\title{
Experimental Study on Impact Force Identification Using Output Response of Polyvinylidene Fluoride Sensor
}

\author{
Cao Vu Dung* and Eiichi Sasaki \\ Department of Civil Engineering, Tokyo Institute of Technology, \\ 2-12-1 Ookayama, Meguro, Tokyo 152-8552, Japan
}

(Received December 27, 2016; accepted June 21, 2017)

Keywords: impact force, identification, PVDF sensor, cantilever beam

Impact force identification from structural dynamic responses at certain points on a mechanical structure subjected to impact is a classical inverse problem in dynamics. There has been a growing demand for an efficient inverse algorithm and/or sensors that can overcome the ill-conditioning problem often encountered in an inverse analysis. This study investigates the capabilities of a polyvinylidene fluoride (PVDF) sensor to identify impact force. Impact force was identified from the response of a PVDF sensor attached to a steel cantilever beam. A simple method involving neither the analytical nor the experimental Green's function was used to create the objective function that was minimized during the optimization process. Results of parametric studies on the effect of PVDF output length included in the process of optimizing the objective function suggest that identification accuracy appears to be more vulnerable to peak differences than to area differences. Output lengths of 50 and $100 \mathrm{~ms}$ appear to yield good identification accuracy for both peak- and area-based evaluations. Furthermore, accuracy of identification seems to be susceptible to variations in the starting time of impact history. However, such susceptibility was overcome by the PVDF sensor's excellent signal-to-noise ratio, thanks to which the starting point of an impact's history can be accurately identified.

\section{Introduction}

Impact force identification from structural dynamic responses such as displacement, velocity, acceleration, or strain at certain points of a mechanical structure or body subjected to impact is a classical inverse problem in dynamics. In many practical situations, direct measurement of external forces acting on an existing vibrating structure is not possible. Instead, structural responses are first measured by appropriate sensors from which the responses are later used to reconstruct the position and magnitude of those external forces. Inverse analysis can be performed by minimizing the error between the measured and predicted data derived from a mathematical model or by training the structure using a neural network. ${ }^{(1)}$ An extensive review of inverse analysis methods for the indirect measurement of impact force can be found in Ref. 2. Previous studies have focused on estimation of impact location and history using different

*Corresponding author: e-mail: caovu@tcu.ac.jp

http://dx.doi.org/10.18494/SAM.2018.1344 
techniques of inverse analysis and different sensor types such as strain gauges, accelerometers, and vibrometers.

Recently, power-generating (passive) sensors have received significant interest for efficient structural health monitoring since power supply to the sensors is not required. Several attempts have been made to utilize sensors made of lead zirconate titanates (PZTs), which is a common type of piezoceramic for impact force identification. Hu et al. ${ }^{(3)}$ proposed an identification technique employing the Chebyshev polynomial to approximate the history of impact force acting on carbon fiber reinforcement polymer laminated plates using embedded PZT sensors. Park et al. ${ }^{(4)}$ presented an inverse method based on a system-identification technique using transfer functions for identifying impact events on a complex structure with built-in piezoelectric sensors without the need of constructing a full-scale accurate structural model of acquiring excessive training data on the structure. Matsumoto et al. ${ }^{(5)}$ proposed a method based on the finite element method and least squares containing a penalty term for the derivative of force history for identifying both location and time of an impact force acting on an aluminum plate using multiple PZT piezoelectric sensor responses. Despite an excellent sensing capability due to relatively high piezoelectric coefficients compared with other piezoelectric materials, PZT sensors exhibit most of the characteristics of ceramics, namely a high elastic modulus, low tensile strength, and brittleness, which makes them less suitable to sensing repeated loading with millions of number of cycles such as fatigue loading.

Sensors using polyvinylidene fluoride (PVDF), a thin film-type polymer that is mechanically tough, flexible, and low density, are considered to be more suitable for structural health monitoring in a fatigue environment such as bridges under repeated vehicular live load. Moreover, Young's modulus of PVDF is approximately 1/12th that of PZT, which makes a PVDF sensor less likely to influence the dynamic of the host structure as a result of its own stiffness in spite of its lower piezoelectric coefficients (approximately 1/10th of PZT). ${ }^{(18)}$ PVDF is a semi-crystalline polymer consisting of long chains with the repeat unit of $\mathrm{CF}_{2} \mathrm{CH}_{2}$, whose crystalline domains appear in four different forms that can be interconverted by the application of heat, electrical fields, and pressure. ${ }^{(6,7)}$ Thermal poling or corona poling orients the molecular dipoles in the crystalline regions and thus yields a permanent polarization. PVDF, when crystallized in its $\beta$-phase, exhibits piezoelectric properties because of which mechanical energy can be converted to electrical energy and vice versa. Therefore, PVDF has been used to manufacture sensors and actuators that can be utilized in various practical applications including shock impact and pressure sensors, ${ }^{(8,9)}$ biomedical sensors, ${ }^{(10,11)}$ acoustic sensors, ${ }^{(12,13)}$ tactile sensors, ${ }^{(14)}$ active vibration control, ${ }^{(15)}$ and structural health monitoring of civil and aerospace structures. ${ }^{(16,17)}$ Sirohi and Chopra ${ }^{(18)}$ demonstrated the superior signalto-noise ratio of PVDF sensors compared to conventional foil gauges for dynamic strain sensing. PVDF sensors proved to be capable of measuring transient responses for dynamic strain, and their sensitivity is better than that of a conventional foil gauge. ${ }^{(19)}$ An attempt to use PVDF sensors for impact force identification was made by Chuang et al., ${ }^{(21)}$ who proposed an experimental method based on the concept of conductivity of metals first proposed by Doyle ${ }^{(20)}$ to demonstrate the capability of PVDF sensors in identifying the dynamic history of impact loadings that were directly applied to the PVDF to obtain the contact time and impact history 
utilizing the PVDF sensor's longitudinal effect, i.e., the impact force is in the same direction as that of the electrical displacement generated in the PVDF sensor.

The information of external forces acting on a structure is of great importance for design and maintenance of civil engineering structures. However, in civil engineering structures it is usually impossible to utilize the PVDF sensor's longitudinal effect because it requires direct contact between the acting force and the sensors. Instead, the in-plane sensing mode, i.e. the contraction and expansion of a PVDF sensor attached to a deformed mechanical host structure's surface, has been frequently employed. In the in-plane sensing mode, PVDF sensors can potentially replace conventional strain gauges in bridge weigh-in-motion (BWIM), a technology that utilizes the responses of sensors attached to structural components of a bridge to identify the dynamic live load induced by vehicles crossing the bridge. Depending on the location of sensors, the vehicle speed, and the length of the influence line (i.e. loading effect), the loading can be extremely short in the time domain for each vehicle passage, which is similar to high strain-rate dynamic impact loading. In current BWIM practice, strain gauges have been mainly employed for sensing in the low strain rate (i.e., low frequency) region. However, strain gauge operation requires continuous power supply and often has a relatively lower signal to noise ratio compared to that of PVDF sensors.

Therefore, this study investigates the capability of PVDF sensors to identify impact force using the sensor's in-plane sensing mode for the efficient long-term monitoring of high strainrate loading induced by vehicles crossing a bridge. For identification of the dynamic live load, the time history of impact load is more desirable than the amplitude because the amplitude can be sensitive to changes in the transverse location of vehicles. This study focuses on identification of the temporal history of an impact force to get accurate variations of the dynamic live load for an enhanced fatigue assessment of bridge deck structures. The transverse location of vehicles is also important but will be addressed in future studies. To obtain a PVDF sensor's transient response for impact force identification, it is practical to apply impact loadings to flexible structures such as a cantilever beam. An impact force applied directly to a cantilever beam can be identified from the transient response of an attached PVDF sensor in an off-line mode. A simple method proposed by $\mathrm{Wu}$ et al. ${ }^{(2)}$ involving neither the analytical nor the experimental Green's function was used to create an objective function that would be minimized during the optimization process. An impact test involving multiple single impacts on a cantilever beam would be conducted to: 1) verify the linear relationship between impact force and output voltage response of the PVDF sensor and 2) generate impact forces that would be identified by the measured response of the PVDF sensor.

\section{Impact Tests}

Impact tests were conducted by inducing impact forces on a steel cantilever beam to which a PVDF sensor was attached (Fig. 1). The cantilever was fixed at one end by the gripping force of the lower chuck of a hydraulic servo so that the cantilever stood in the vertical direction. The consistency of impact location was controlled using a 2-mm radius circle mark centering at the desired impact location. Any impact leaving an imprint outside the circle was eliminated. A 


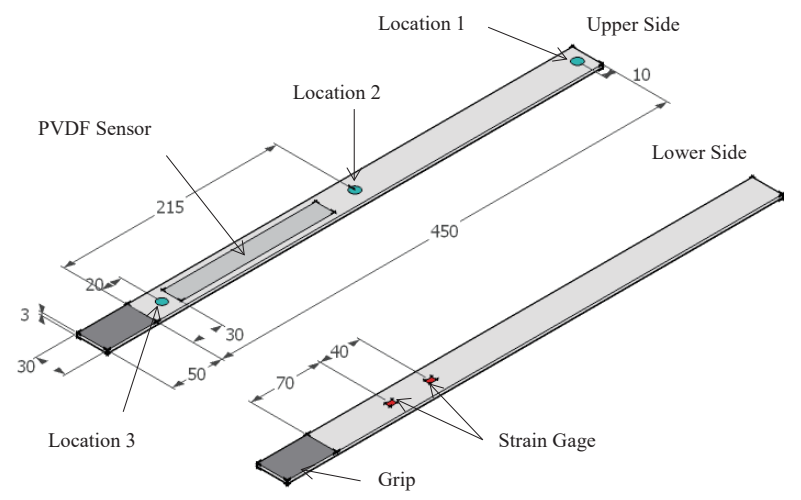

Fig. 1. (Color online) Cantilever beam with attached sensor and impact locations.

piezo film lab amplifier ${ }^{(23,24)}$ (herein, charge amplifier) with a 0.01 to $1000 \mathrm{mV} / \mathrm{pC}$ sensitivity range in charge-mode operation was used for signal conditioning during the impact test. A feedback capacitance of $100 \mathrm{nF}$ was selected. The charge amplifier provided options for multipole, low-pass, and high-pass filters with $-3 \mathrm{~dB}$ frequencies ranging from 0.1 to $100 \mathrm{kHz}$. The lower and higher cut-off frequencies were selected as 0.1 and $100 \mathrm{kHz}$, respectively. For data acquisition, a NI USB-4431 that enabled four 24-bit simultaneous analog inputs was used. The sampling rate was set at $10 \mathrm{kS} / \mathrm{s}$, and a $1 \mathrm{kS} / \mathrm{s}$ data windowing was chosen for realtime visualization of the impact signal during the test. A DT4-028 K/L PVDF sensor ${ }^{(24)}$ was attached to a steel cantilever beam by commercial double-coated adhesive tape so that one edge of the PVDF sensor was at a distance of $30 \mathrm{~mm}$ from the fixed end of the cantilever beam (Fig. 1). A modally tuned impact hammer PCB 086C03 manufactured by PCB Piezotronics with an attached plastic tip was used to manually generate impact forces with different magnitudes on the cantilever beam at the three impact locations illustrated in Fig. 1. During the impact test, impact signals sensed by the tip of the hammer were transmitted to an integrated circuit piezoelectric (ICP) sensor signal conditioner provided by PCB Piezotronics. Strain gauges were also attached to the opposite surface of the cantilever beam. The responses of strain gauges were regarded as "true strain" that was used to calibrate the impact forces (converting voltage to force units) using finite element analysis of the cantilever beam.

\section{Relationship of Impact Force to PVDF Output}

To investigate the relationship between impact force and output voltage of a PVDF sensor attached to the cantilever beam, multiple impact forces with varying amplitudes were applied at the three locations illustrated in Fig. 1. Two methods were employed to evaluate the impact force and the PVDF sensor's output voltage. The first was a peak-based method, in which the peak of the first cycle of both the impact force and the resulting output signal of the PVDF sensor were evaluated. Meanwhile, the second method was an energy-based evaluation using the area under the curve of the first cycle. Typical impact force and PVDF sensor's output voltage for all three locations are shown in Fig. 2. Impact histories at locations 1 and 

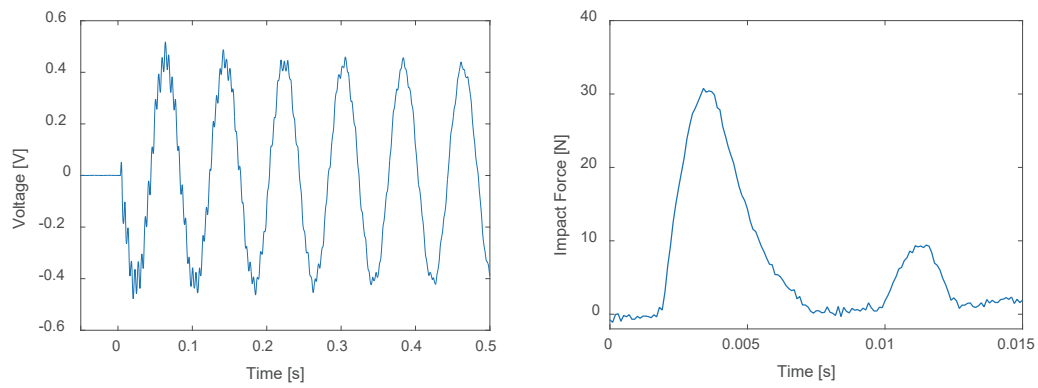

(a)
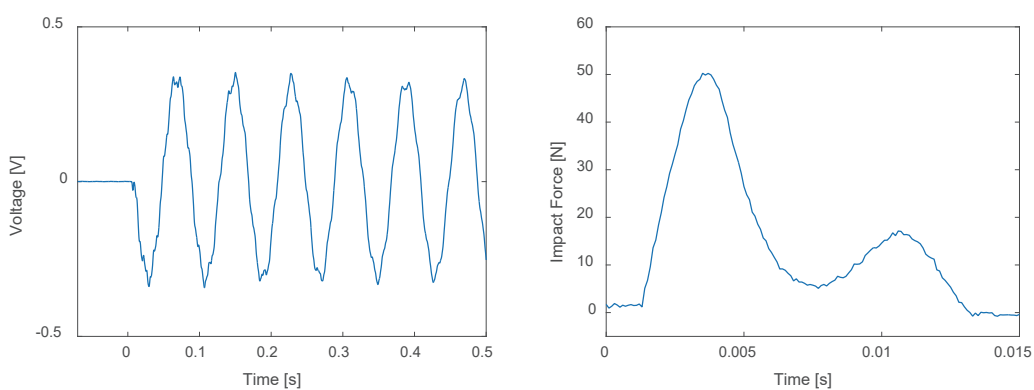

(b)
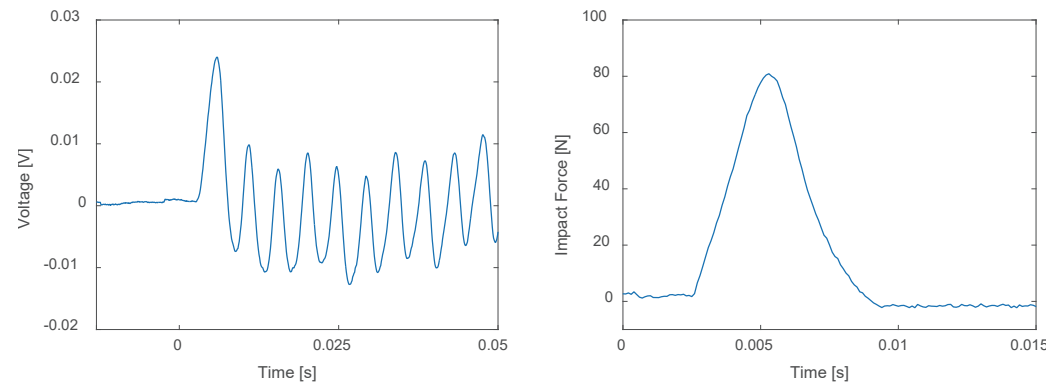

(c)

Fig. 2. (Color online) Typical impact test results for impacts at all three locations. (a) Location 1, (b) location 2, and (c) location 3.

2 contain two peaks that are both due to the physical contact between the impact hammer and the cantilever beam. The first peak represents the first time when the cantilever beam was hit by the hammer tip. The energy absorbed by the cantilever beam is then transformed to displacement of the cantilever beam's free end, which mainly excites the first vibration mode. Right after the first collision, the hammer tip continues to move in the same direction as that of the cantilever beam's free end due to the effect of inertia induced by the hammer's weight and velocity. Approximately one to two milliseconds after the first collision, the hammer hits the tip of cantilever beam the second time, which causes the second impact. However, for impact at location 3 which is close to the fixed end, impact energy absorbed by the cantilever beam is transformed mainly into excitation of higher modes rather than displacement at the point of collision. Therefore, only one peak appears in the time history of impact at location 3. Linear curve-fitting was applied to verify a linear relationship between impact force and output 
voltage (Fig. 3). The results of linear curve-fitting (Table 1) indicate that a linear relationship exists between impact force and output voltage of a PVDF sensor for both evaluation methods. However, the strength of the linear relationship seems to be lower for impact at location 3 compared with those for locations 1 and 2. It should be noted that the amplitude of the output voltage for impacts at location 3 is much lower than those for locations 1 and 2; hence values for 3 might have been more vulnerable to any small discrepancy in impact position.

\section{Impact Force Identification}

The objective of this study was to identify the time history of an impact force using the output voltage of a PVDF sensor attached to a cantilever beam. A method reported by Wu et $a l .{ }^{(22)}$ using signals obtained directly from an instrumented impact hammer was used, in which
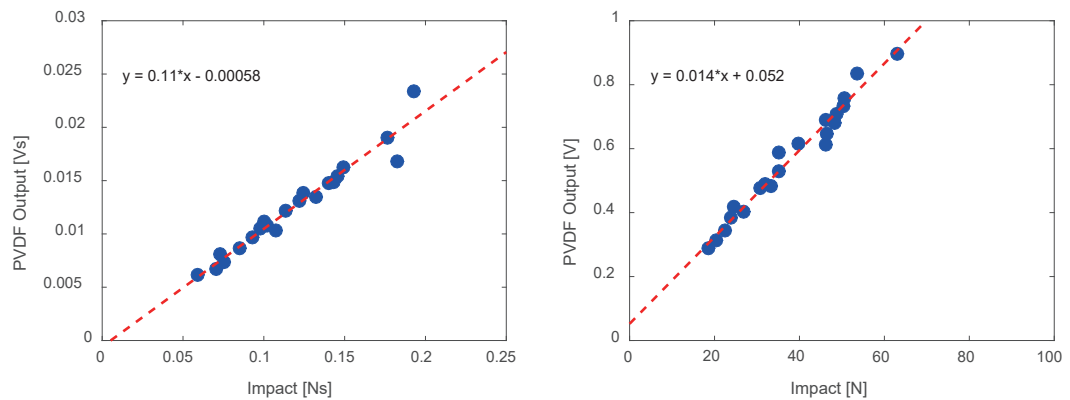

(a)
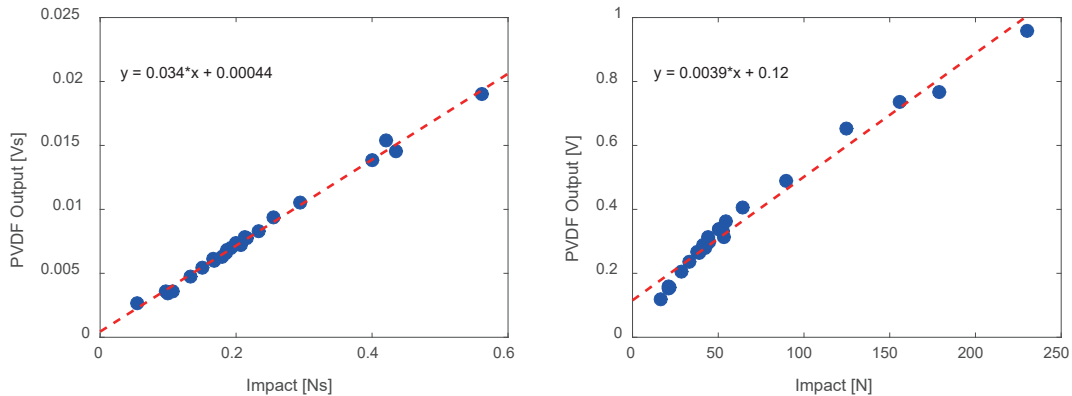

(b)
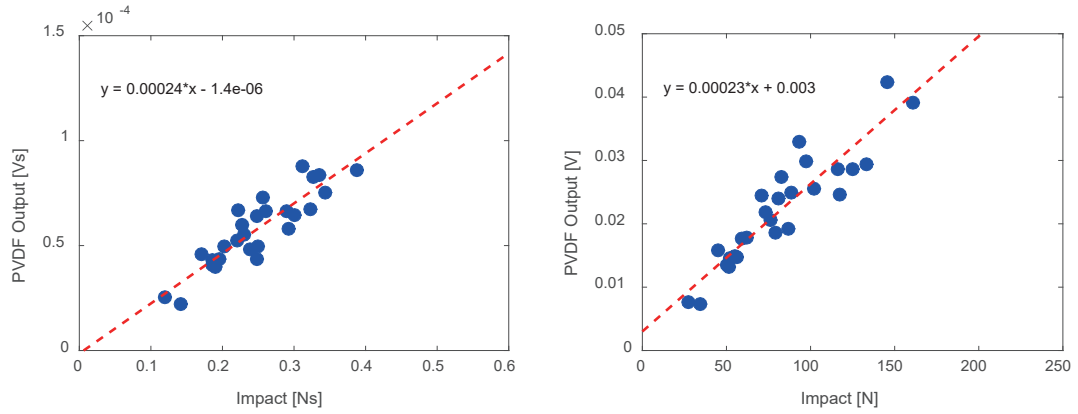

(c)

Fig. 3. (Color online) Linear curve-fitting for data of impacts at three locations. (a) Location 1, (b) location 2, and (c) location 3. 
Table 1

Linear curve-fitting results for area- and peak-based methods.

\begin{tabular}{llll}
\hline Area method & Location 1 & Location 2 & Location 3 \\
\hline Regression model & & $\mathrm{y} \sim 1+\mathrm{x} 1$ & \\
No. of observations & 21 & 23 & 27 \\
Error DOF & 19 & 21 & 25 \\
Intercept & 0.00057726 & -0.0004429 & $-1.4335 \mathrm{e}-06$ \\
$\mathrm{x} 1$ & -0.11055 & -0.033608 & 0.00023851 \\
RMS error & 0.000982 & 0.000297 & $7.93 \mathrm{e}-06$ \\
$R$-squared & 0.951 & 0.995 & 0.8 \\
$p$-value (F-test) & $7.15 \mathrm{e}-14$ & $8.77 \mathrm{e}-26$ & $3.17 \mathrm{e}-10$ \\
\hline Peak method & Location 1 & Location 2 & Location 3 \\
\hline Regression model & & $\mathrm{y} \sim 1+\mathrm{x} 1$ & \\
No. of observations & 21 & 23 & 27 \\
Error DOF & 19 & 21 & 25 \\
Intercept & -0.052327 & -0.11564 & 0.003005 \\
x1 & -0.013569 & -0.0038636 & 0.000233 \\
RMS error & 0.0296 & 0.0334 & 0.0035 \\
$R$-squared & 0.972 & 0.977 & 0.842 \\
$p$-value (F-test) & $2.97 \mathrm{e}-16$ & $9.51 \mathrm{e}-19$ & $1.68 \mathrm{e}-11$ \\
\hline
\end{tabular}

the force history induced in a second impact event with an unknown profile could be detected while involving neither the analytical nor the experimental Green's function. The relationship between an impact force and the output response for a general structure can be written as:

$$
R_{r e f}=G^{*} P_{r e f}
$$

where * means convolution in the time domain; $R_{r e f}$ and $P_{r e f}$ are the output voltage of the PVDF sensor and the impact force recorded by the impact hammer, respectively, both of which are known values for a reference impact event; $G$ is Green's function.

When the structure is hit by the impact hammer again at the same location by an unknown impact force $P$ and a known output response $R$, the convolution relationship becomes

$$
R=G^{*} P .
$$

Since the commutative law holds for convolution, the following expression can be derived:

$$
R * P_{r e f}=\left(G * P_{r e f}\right) * P
$$

Finally, the following convolution relationship is obtained,

$$
R^{*} P_{r e f}=R_{r e f} * P,
$$

which indicates that the impact force history $P$ can be obtained without knowledge of Green's function for a given set of known $R, R_{r e f}$, and $P_{\text {ref }}$ values. 
An objective function was defined using MATLAB's fmincon function to find the minimum of a constrained nonlinear multivariate function $f(x)$ such that:

$$
\left\{\begin{array}{l}
c(x) \leq 0 \\
c e q(x)=0 \\
A \cdot x \leq b \\
\text { Aeq. } x=b e q \\
l b \leq x \leq u b .
\end{array}\right.
$$

The fmincon function (fun, $x 0, A, b, A e q, b e q$ ) starts at $x 0$ and attempts to find a minimizer $x$ of the function described in fun subject to the linear inequalities $A^{*} x \leq b$ until a local minimum is found that satisfies the constraints and optimization is completed because the objective function is non-decreasing in feasible directions, to within the default value of the optimality tolerance, and constraints are satisfied to within the default value of constraint tolerance.

The following objective function for the optimization procedure was defined:

$$
\begin{aligned}
E & =\frac{1}{2}\left\|R * P_{r e f}-R_{r e f} * P\right\|^{2}, \\
P & \geq 0 \\
P_{(t=0)} & =0 .
\end{aligned}
$$

In the report by $\mathrm{Wu}$ et al., ${ }^{(22)}$ an optimization procedure using the gradient projection method was proposed to solve Eq. (2) to overcome the problem of non-convergence of conventional methods, such as Gaussian elimination, due to the ill-conditioned problem of Green's function. The authors reasoned that truncated errors are induced in the discretization process of Green's function, and the signals recorded in an experiment are always contaminated with noise. For the same reasons, gradient projection method was also used to solve Eq. (4) to search for the optimal solution of $P$. By using a similar optimization procedure, convergence to a local minimum was achieved in the current study.

Figure 4 shows a typical reference impact event and the arbitrary impact event for which the impact force history is to be identified. The identified impact force is plotted against the actual value in Figs. 5-7.

\subsection{Verification by numerical simulation}

The identified impact force was verified by comparing the PVDF sensor output voltage responses obtained in the experimental impact test with those obtained by simulation using the obtained impact history. Numerical simulation was carried out using the commercial software MATLAB. It was demonstrated that most of the flexural modes can be accurately identified by the PVDF sensor, which indicates the capability of the PVDF sensor to identify the natural frequencies of the host structure's fundamental vibration modes from free vibration responses. Direct damping ratios determined by the half-power point method using the frequency response 

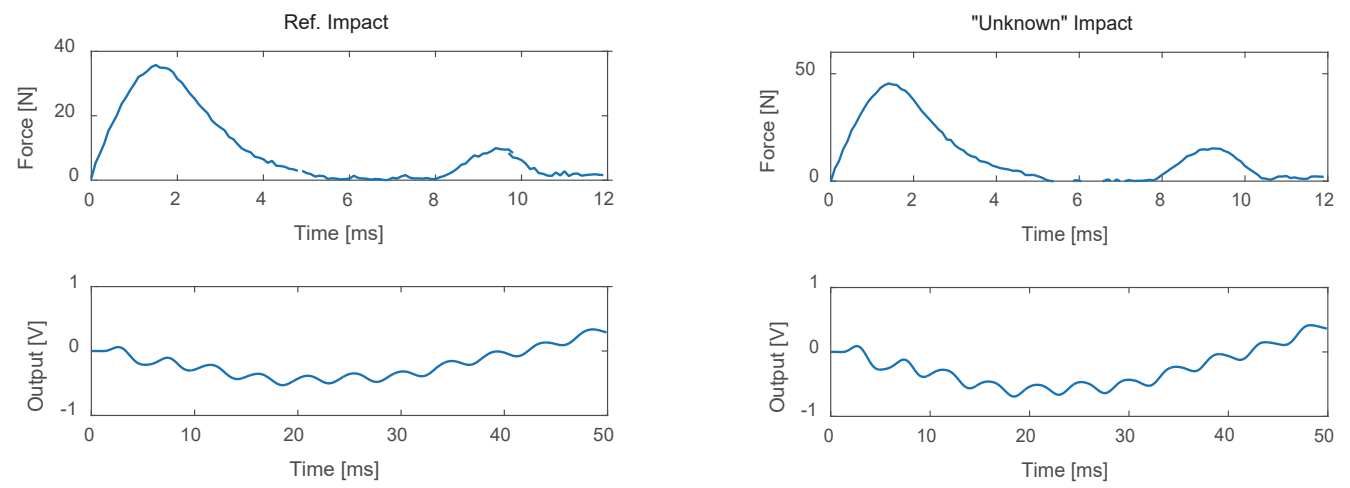

(a)

(b)
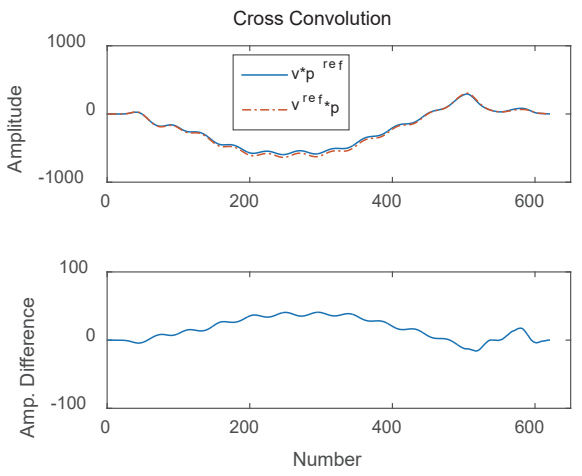

(c)

Fig. 4. (Color online) Measured impact force and PVDF sensor's voltage output of (a) reference impact event, (b) an arbitrary impact event for which the impact force is to be identified, and (c) cross convolution, for impact location 1.

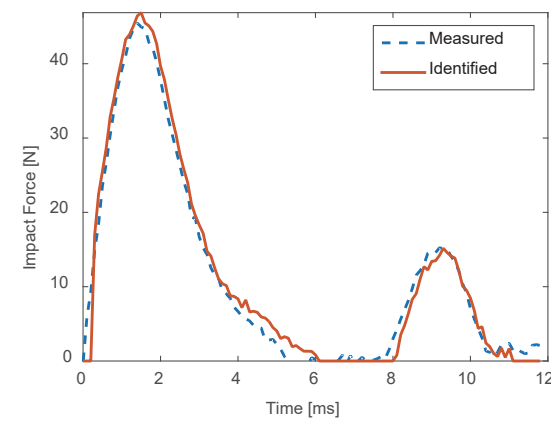

Fig. 5. (Color online) Typical identified impact forces for impact at location 1 .

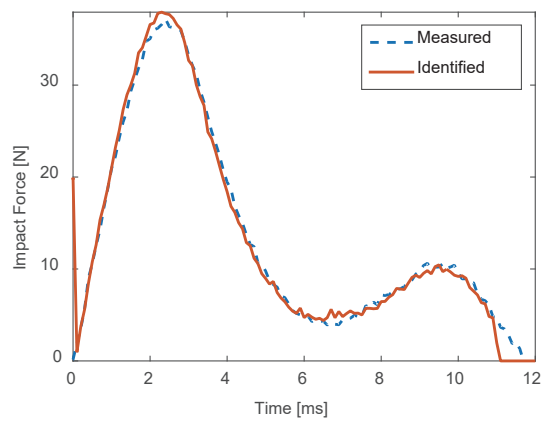

Fig. 6. (Color online) Typical identified impact forces for impact at location 2 .

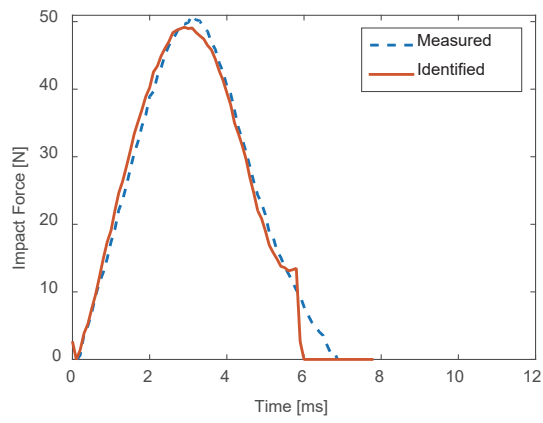

Fig. 7. (Color online) Typical identified impact forces for impact at location 3. 
functions shown in Fig. 8 were used for the first five flexural modes of the cantilever beam in the FEM analysis (Table 2). Figure 9 shows a reasonable agreement between simulated and experimental responses of a PVDF sensor.

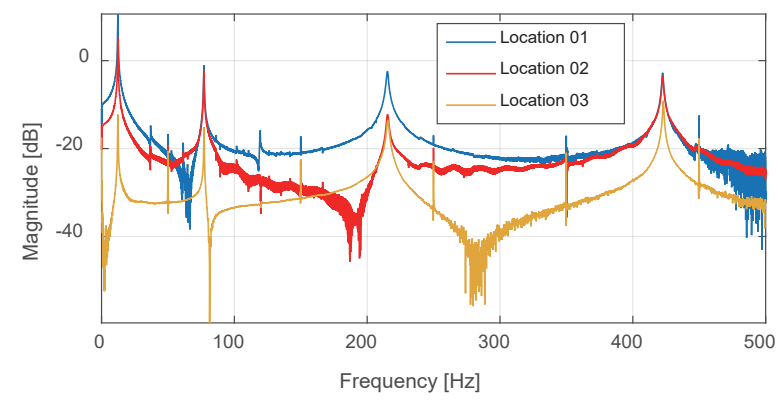

Fig. 8. (Color online) Frequency response functions for three impact locations.

Table 2

Identified damping ratio by half-power method.

\begin{tabular}{lccccc}
\hline & 1st mode & 2nd mode & 3rd mode & 4th mode & 5th mode \\
\hline Impact at 01 & 0.011159 & 0.002566 & 0.005434 & - & - \\
Impact at 02 & 0.009391 & 0.002647 & 0.006121 & - & - \\
Impact at 03 & 0.013542 & 0.002346 & 0.005920 & 0.001362 & 0.002831 \\
\hline
\end{tabular}

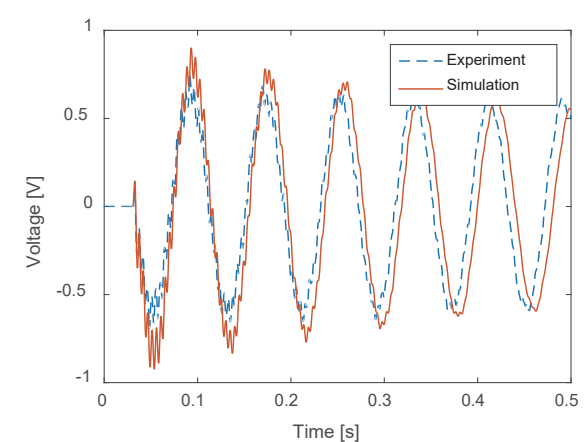

(a)

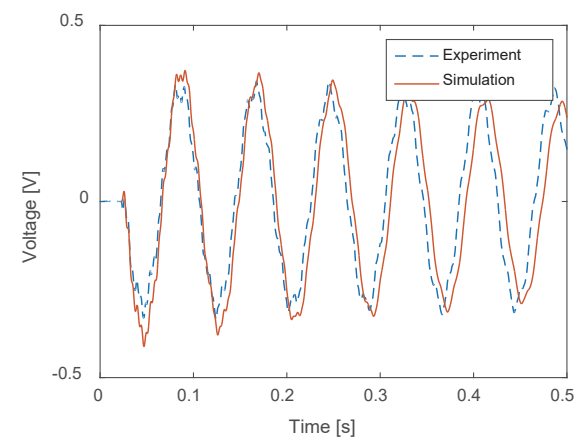

(b)
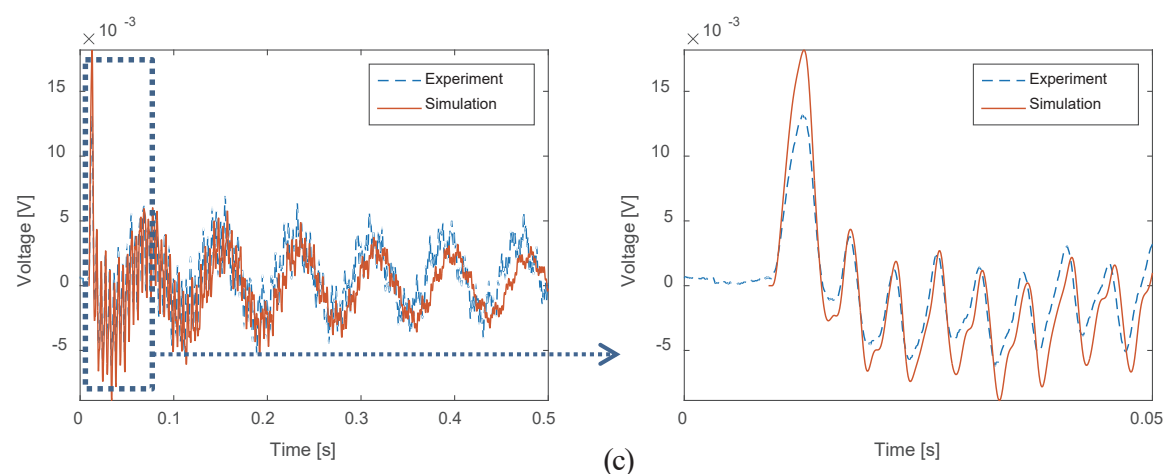

Fig. 9. (Color online) Verification of identified impact using simulated output response of PVDF sensor. Impact for (a) location 1, (b) location 2, and (c) location 3. 


\subsection{Effect of PVDF sensor's output length}

The effect of length of PVDF output voltage signal included in the deconvolution process on the identified impact history was evaluated by varying the length of the PVDF output signal from 0.01 to $1 \mathrm{~s}$ and 0.002 to $0.1 \mathrm{~s}$ for locations 1 and 2 and location 3 , respectively. The ability to accurately identify impact was evaluated by both peak and area differences. The peak difference was evaluated by comparing both values of peaks for locations 1 and 2 and the value for one peak for location 3 between the measured impact and the impact estimated by each PVDF output length value used in the optimization process. Figures 10 and 11 indicate that, for impact at locations 1 and 2, identification accuracy is more vulnerable to peak difference than to area difference. In most cases, with appropriate output length of 50 or $100 \mathrm{~ms}$, identification accuracy is around $5 \%$ in terms of area difference, while peak difference yields an accuracy of less than 10\%. For location 3, an output length of 10-100 ms was found to result in about 10 and $5 \%$ for peak and area difference, respectively (Fig. 12).

\subsection{Effect of starting time of output response}

In the calculation of cross convolution, the starting point of the PVDF sensor's output response, i.e., point " $0 \mathrm{~ms}$ " on the time axis in Figs. 2 and 4 , was determined by visual observation thanks to the PVDF sensor's high signal-to-noise ratio. To evaluate the effect of the output response's starting time on the identified impact signal, five different starting time

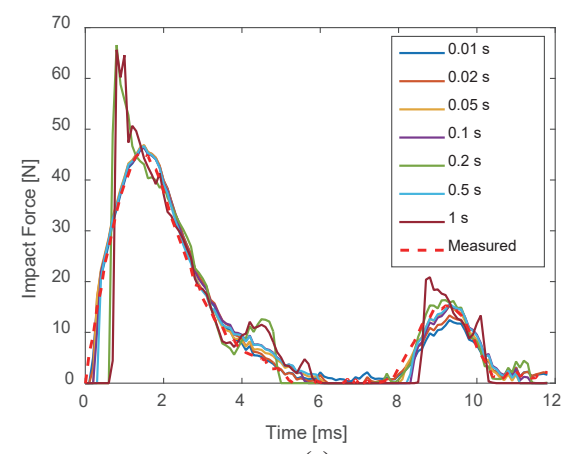

(a)

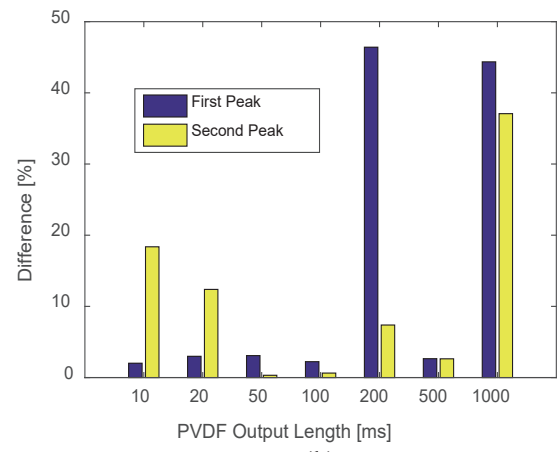

(b)

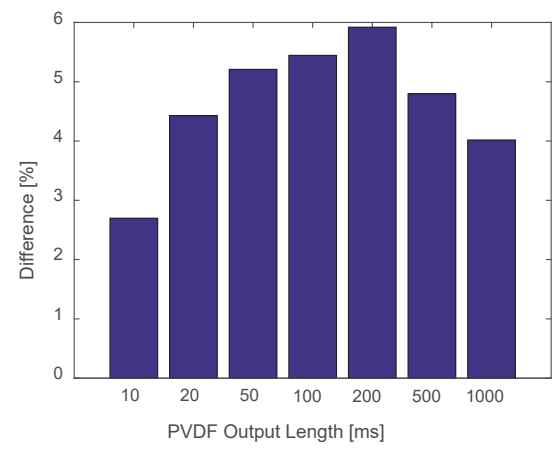

(c)

Fig. 10. (Color online) Effect of output length for a typical impact at location 1. (a) Identified impact forces, (b) peak difference, and (c) area difference. 


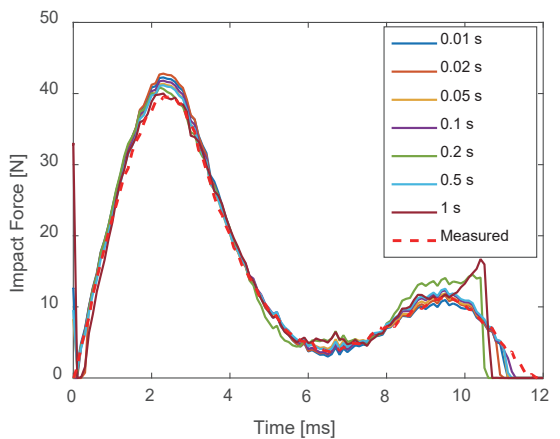

(a)

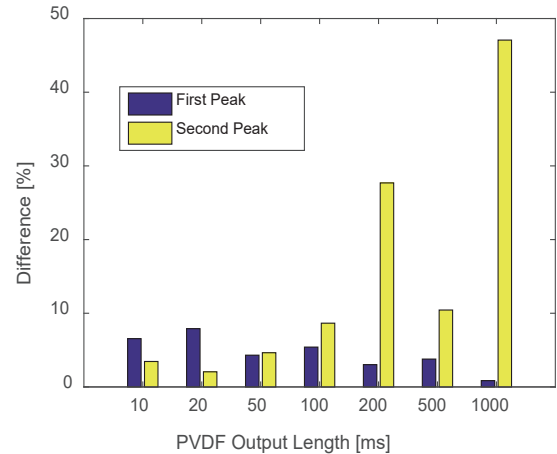

(b)

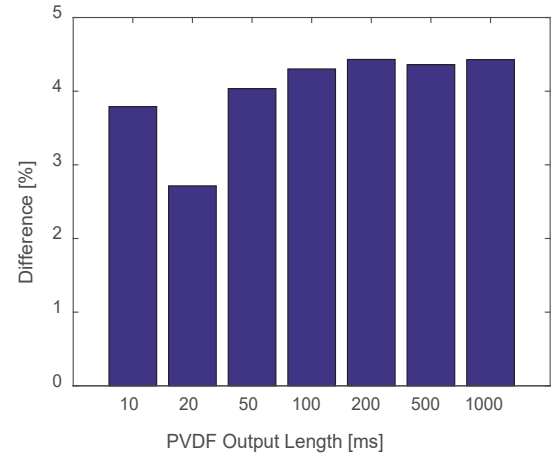

(c)

Fig. 11. (Color online) Effect of output length for a typical impact at location 2. (a) Identified impact forces, (b) peak difference, and (c) area difference.

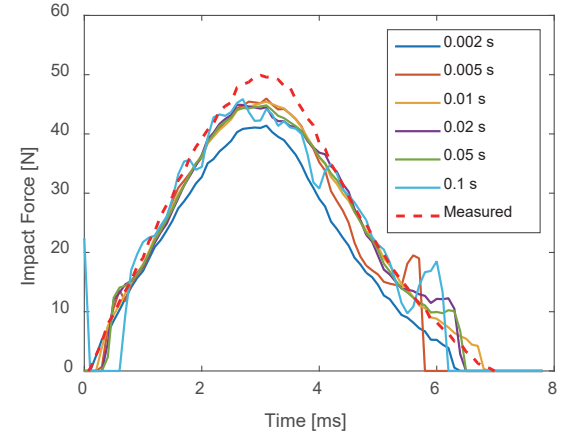

(a)

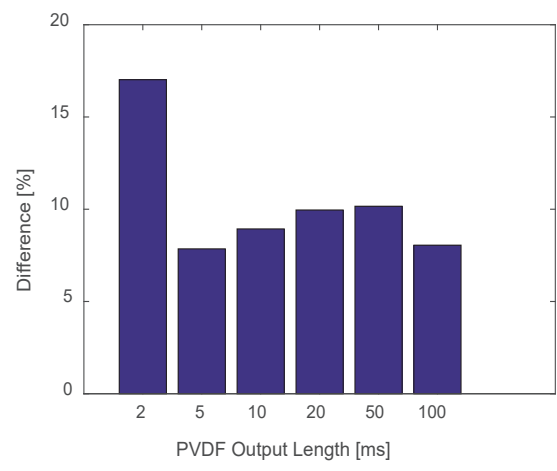

(b)

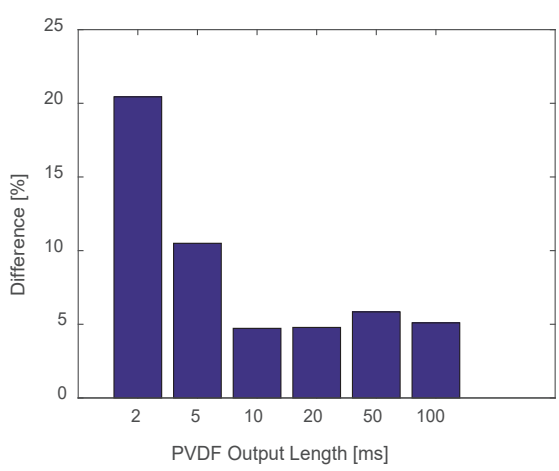

(c)

Fig. 12. (Color online) Effect of output length for a typical impact at location 3. (a) Identified impact forces, (b) peak difference, and (c) area difference. 
delays were selected, including $-1,-0.5,0,0.5$, and $1 \mathrm{~ms}$. The effect of starting time appears to be significant since a variation of only $1 \mathrm{~ms}$ lead to a significant increase in peak difference, i.e., decrease in accuracy, from less than $10 \%$ to more than 20\% [Fig. 13(b)]. Although variations in starting time appear to affect the difference in area less, these result in large observed phase differences [Figs. 13(a) and 13(c)]. However, thanks to the excellent signal-to-noise ratio of the PVDF sensor, the actual starting point of contact (i.e., the starting point of impact force history) can be accurately identified.

\section{Discussions}

If the output length is too short, it will not provide sufficient and reliable input for optimization. On the other hand, if it is too long, it will magnify the difference between arbitrary and reference responses, decreasing the accuracy. A sensor output length of 50-100 ms appears to be an optimal selection that leads to reasonable accuracy for all impact locations. Accuracy of identification seems to be susceptible to the variations in the starting time of the impact history included in the optimization process. However, such susceptibility can be overcome by PVDF sensors' excellent signal-to-noise ratio, thanks to which the starting point of an impact history can be accurately identified. The findings of this study indicate that PVDF sensors can be employed to identify the temporal history of impact force using the most fundamental structure, which is a cantilever beam, and a simple identification approach by $\mathrm{Wu}$

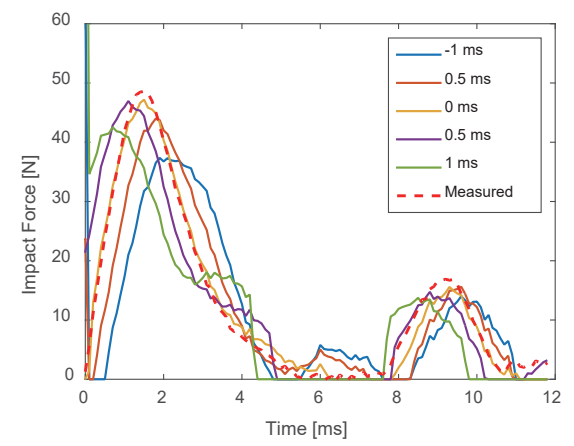

(a)

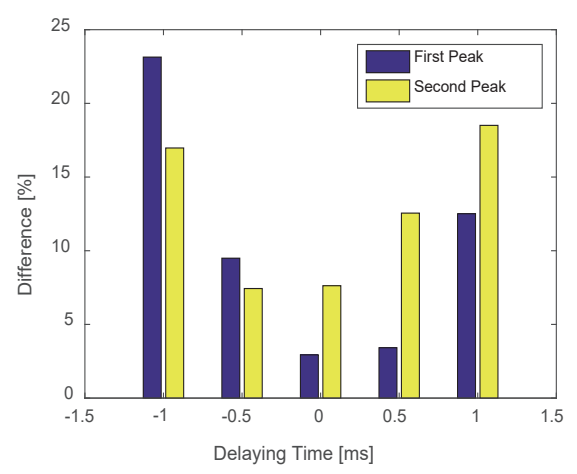

(b)

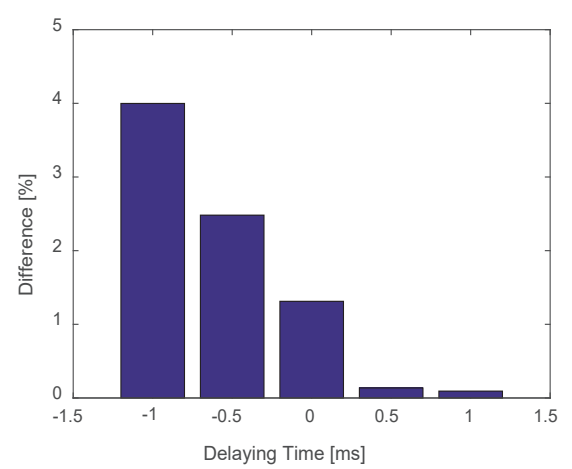

(c)

Fig. 13. (Color online) Effect of starting time of output response for impact at location 1. (a) Identified impact forces, (b) peak difference, and (c) area difference. 
et al. ${ }^{(22)}$ utilizing the concept of Green's function that is assumed to be a constant for a linear system. For identification of a moving vehicular live load on a bridge deck, the impact location in both the bridge axis and transverse directions and the magnitude vary continuously due to the dynamic interaction between a vehicle and the bridge deck. Therefore, future studies should investigate the capability of PVDF sensors to identify both location and magnitude of a moving impact force on a 2D structure using an identification method that does not rely on a reference impact force and sensor response.

\section{Conclusions}

Impact tests were conducted on a cantilever beam to which a PVDF sensor was attached to investigate the relationship between output voltage and impact force at three different locations and the capability of PVDF sensors to identify impact force. Two evaluation methods, one peakand one energy-based, were employed for the evaluation of the relationship between output voltage and impact force. The impact test results indicated that a significant linear relationship exists between a PVDF sensor's output voltage and impact force for both evaluation methods. A simple method involving neither the analytical nor the experimental Green's function was employed to define the objective function that would be minimized during the optimization process to identify the impact force. A good agreement between measured and identified impact history was observed for all three impact locations. The identified impact was verified by the PVDF sensor's output voltage response obtained by simulation. Results of parametric studies on the effect of PVDF output length included in the optimization process function suggest that identification accuracy appears to be lower when evaluated by peak difference than by area difference for impact at locations 1 and 2. PVDF sensors' output lengths of 50 or $100 \mathrm{~ms}$ appear to yield good identification accuracy for all impact locations. The identification results indicate that PVDF sensors can be employed to identify the temporal history of impact force. However, future studies are needed to extend the contribution of this study to identify both time history and location of a moving impact force on a more complicated structure.

\section{References}

1 S. Ahmari and M. Yang: Smart Mater. Struct. 22 (2013) 085024.

2 H. Inoue, J. J. Harrigan, and S. R. Reid: Appl. Mech. Rev. 54 (2001) 6.

3 N. Hu, H. Fukunaga, S. Matsumoto, B. Yan, and X. H. Peng: Int. J. Impact Eng. 34 (2007) 1258.

4 J. Park, S. Ha, and F. K. Chang: AIAA J. Air Transp. 47 (2009) 9.

5 S. Matsumoto, M. Tajima, and H. Fukunaga: JSCE 22 (2005) 2.

6 G. M. Sessler: J. Acoust. Soc. Am. 70 (1981) 6.

7 N. Darasawa and W. A. Goddard: Macromolecules 25 (1992) 7268.

8 F. Bauer: 11th Int. Symp. Electrets (IEEE, 2002) 219.

9 A. V. Shirinov and W. K. Schomburg: Sens. Actuators, A 142 (2008) 49.

10 K. J. Kim, Y. M. Chang, S. Yoon, and H. J. Kim: Integr. Ferroelectr. 107 (2009), 53.

11 Y. Chiu, W. Lin, H. Wang, S. Huang, and H. Wu: Sens. Actuators, A 189 (2013), 328.

12 J. Xu: Microphone Based on Polyvinylidene Fluoride (PVDF) Micro-pillars and Patterned Electrodes. Doctoral Dissertation, Ohio State University (2010).

13 J. Xu, L. M. Headings, and M. J. Dapino: IEEE Sens. J. 15 (2015) 5.

14 L. Seminara, M. Capurro, P. Cirill, G. Cannata, and M. Valle: Sens. Actuators, A 169 (2011) 49. 
15 C. Ma, K. Chuang, and S. Pan: IEEE Trans. Ultrason. Ferroelectr. Freq. Control 12 (2011) 2539.

16 E. Matsumoto, S. Biwa, K. Katsumi, Y. Omoto, K. Iguchi, and T. Shibata: NDT\&E Int. 37 (2004) 57.

17 M. Kurata, X. Li, K. Fujita, L. He, and M. Yamaguchi: Sensors and Smart Structures Technologies for Civil, Mechanical, and Aerospace Systems, Proc. SPIE 8692 (2013) 86920F-1.

18 J. Sirohi and I. Chopra: J. Intell. Mater. Syst. Struct. 11 (2000) 246.

19 C. Ma, Y. Huang, and S. Pan: Sensors 12 (2012) 2088.

20 J. F. Doyle: Exp. Mech. 27 (1987) 229.

21 K. C. Chuang, C. C. Ma, and C. K. Chang: Exp. Mech. 54 (2014) 483.

22 E. Wu, T. D. Tsai, and C. S. Yen: Exp. Mech. 35 (1995) 11.

23 Measurement Specialties Inc.: http://www.meas-spec.com (accessed April 2015).

24 Tokyo Sensor: http://www.t-sensor.co.jp/en/ (accessed April 2015). 